\title{
TBXT Gene Duplication
}

National Cancer Institute

\section{Source}

National Cancer Institute. TBXT Gene Duplication. NCI Thesaurus. Code C83916.

Duplication of the T gene which plays an important role in notochord development. This genetic abnormality is implicated in the pathogenesis of familial chordoma. 